\title{
A Low Cost Approach to Specimen Level Imaging of Natural History Microscope Slides using a DSLR System
}

\author{
E. Louise Allan ${ }^{1 *}$, Benjamin W. Price ${ }^{1}$, Olha Shchedrina ${ }^{1}$, Steen Dupont ${ }^{1}$, Laurence Livermore ${ }^{1}$, \\ and Vince S. Smith ${ }^{1}$ \\ ${ }^{1}$ Natural History Museum, Cromwell Road, SW7 5BD, London \\ ${ }^{*}$ Corresponding author: louise.allan@nhm.ac.uk
}

\section{Abstract}

Specimen level imaging of microscope slides often uses automated digital microscopy systems, however, these systems are not always suitable for non-standard slides, which are frequently found in natural history collections. Currently, for these types of slides imaging requires the use of less automated alternatives. This paper presents a low cost option for imaging non-standard slides, by using a DSLR camera with a 1:1 - 5:1 macro lens mounted to a macro focusing rail and a flashbox. There was no noticeable difference in the image resolution between this DSLR-based system and the $5 x$ magnification provided using an automated slide scanner. The DSLR-StackShot system, while only partially automated, enables the required flexibility and manual control required when imaging slides of varying sizes, thicknesses, and preservation types, as well as slides that are damaged or in poor condition.

\section{Keywords}

Microscope slides, natural history collections, specimen digitisation, DSLR

\section{Background}

Digitisation of specimens enables access to natural history collections for new audiences and improves research opportunities (Drew et al. 2017; Decker et al. 2018). In 2014 the Natural History Museum, London (NHM) embarked on an ambitious Digital Collections Programme (DCP) to digitise its collections, estimated to comprise 80 million specimens. One of the aims of the DCP is to develop digitisation workflows for each collection type. For slide mounted specimens two digitisation approaches can be utilised, one approach focuses on capturing an overview image of the slide and its associated labels (Heerlien et al. 2015; Allan et al. 2019; Summerfield et al. in prep), while the other focuses on specimen level imaging of the slide mounted material (Rojo et al. 2006; Musson et al. 2015; Summerfield et al. in prep). 
Overview imaging of microscope slides can be carried out using simple systems and can be used for mass digitisation of collections to extract specimen data (Heerlien et al. 2015; Allan et al. 2019). Specimen level imaging on the other hand requires digital microscopy systems (Rojo et al. 2006). Automated slide digitisation systems, designed for higher resolution imaging of standardised material, have existed for over a decade but have been confined to medical slides with no largescale digitisation projects of natural history slides known to us (Rojo et al. 2006, Weinstein et al. 2009, Dietrich et al. 2012). While there have been several pilot projects that have used specially modified histology slide scanners adapted for natural history specimens, they currently cannot accommodate damaged slides, or slides with non-standard thickness or length (Musson et al. 2015; Summerfield et al. in prep) - issues that can be frequent in natural history collections (Figure 1).
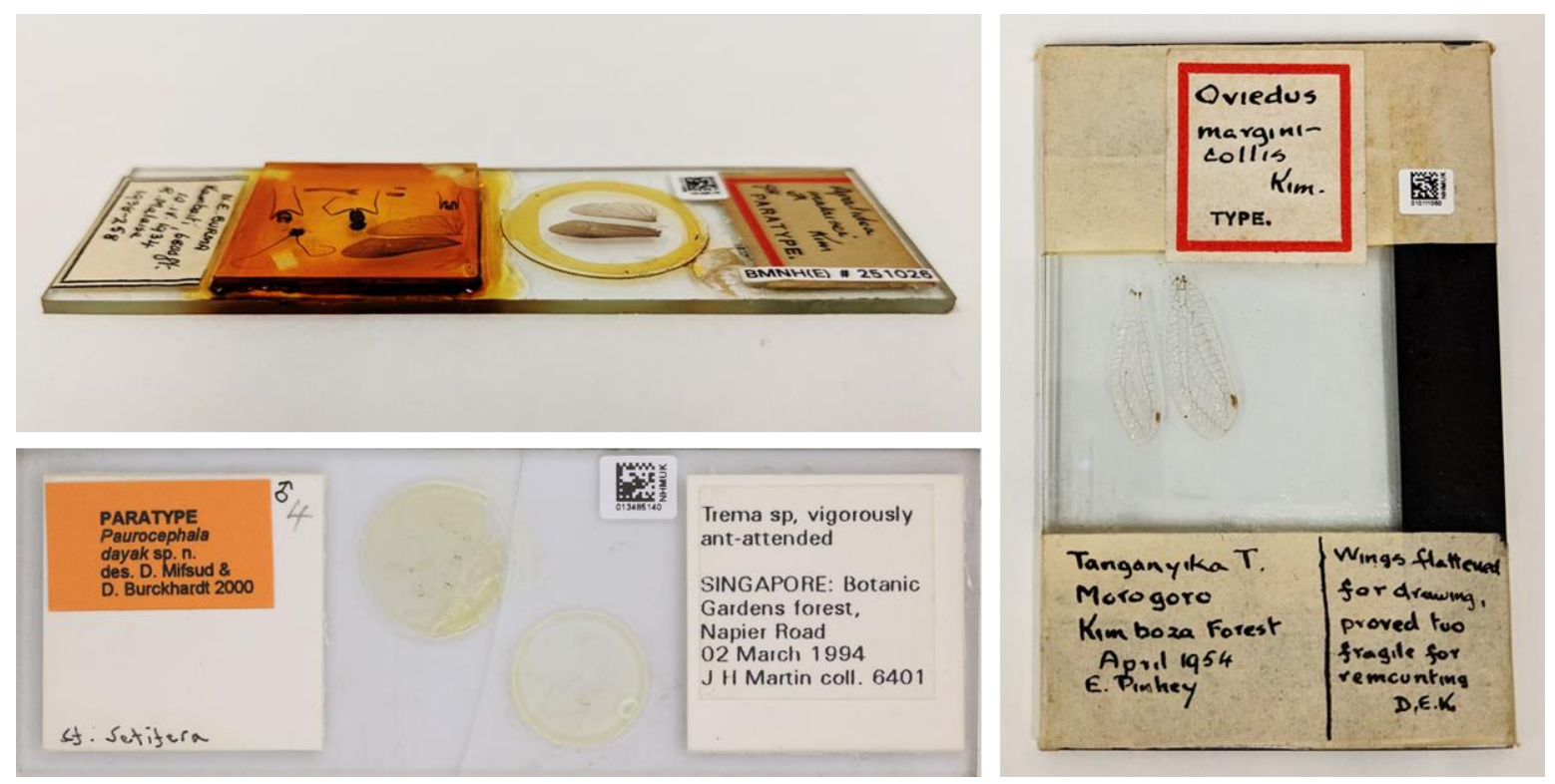

Figure 1. Examples of natural history microscope slides that are damaged or non-standard in size and mountant thickness.

The NHM holds an estimated 2.4 million microscope slides in its collection. These slides are distributed across diverse curatorial groups (e.g. botany, entomology, mineralogy, palaeontology and zoology), with each group having its own distinct slide preparation techniques. In 2015 the DCP conducted a pilot project to digitise a variety of slide mounted material using a specially modified histology slide scanner, the ZEISS Axio Scan.Z1 (Summerfield et al. in prep). This slide scanner is a highly automated system that can image up to a 100 slides per run (Figure 2); however, it is designed to image modern standardised slides and thus adaptations were required to enable the digitisation of natural history slides. Some slides, however, could not be imaged using an automated slide scanner i.e. damaged slides or those with thick mountants, and had to be imaged using a less automated system, such as the Axio Zoom.V16 (Summerfield et al. in prep). 

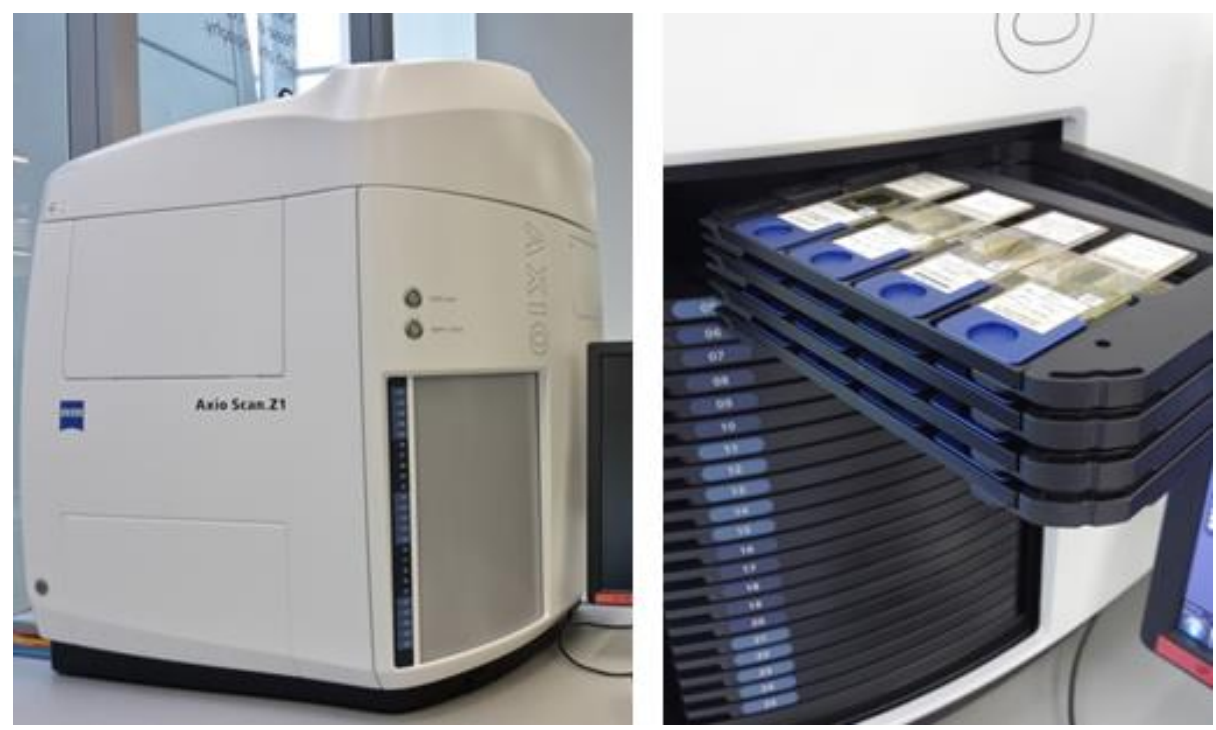

Figure 2. Axio Scan.Z1 is an automated slide scanner that uses magazines to move the slides into position for imaging and can image up to 100 slides per run.

In 2017 the DCP digitised the microscope slide collection of Phthiraptera (Allan et al. 2019), of which one of the aims was to specimen level image a representative of each species, focusing on type material where present. A proportion of the slides (16\%) were unsuitable for the automated slide scanner and were imaged using a Canon EOS 5DS R with a MP-E 65mm lens, StackShot Macro Rail system (Cognisys Inc.), and a custom flashbox (Figure 3). This customised setup enabled us to adapt our existing whole slide imaging setup, described in Allan et al. (2019), thus creating a low cost alternative for specimen level imaging of microscope slides.
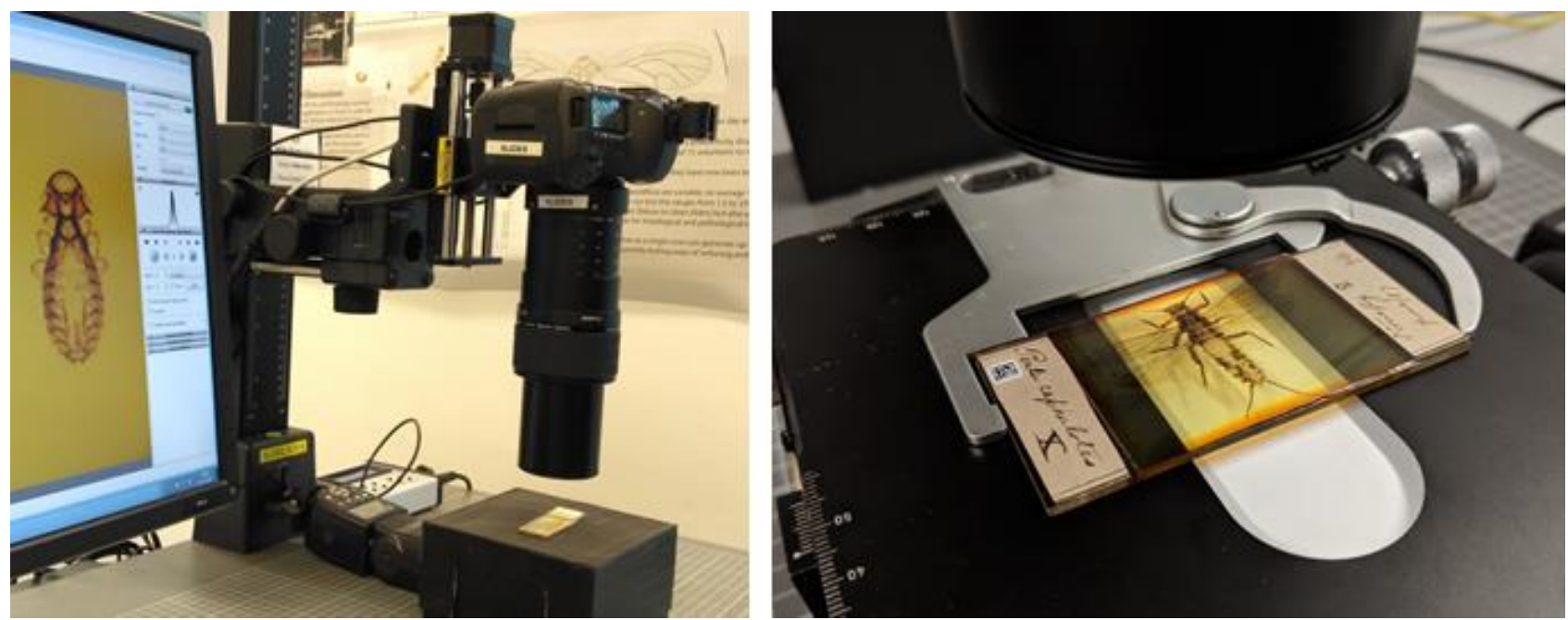

Figure 3. DSLR-StackShot system uses a DSLR camera vertically mounted to a StackShot Macro Rail with a custom flashbox and microscope stage. 


\section{DSLR-StackShot System}

\section{Hardware}

A DSLR camera (Canon EOS 5DS R), with a 1:1 - 5:1 macro lens (Canon MP-E 65mm), was mounted on the Stackshot Macro Rail and attached to a copy stand in a vertical orientation (Figure 3). A custom built flashbox was placed on the copy stand, directly under the lens, and was fitted with a decommissioned microscope stage to enable fine-scale movement (Figure 3). A flash (YongNuo YN-560 III) was inserted into the opening in the flashbox and tethered to the camera with an extension cable. The camera was set to manual mode, f1/5.6, ISO 100, shutter speed $1 / 200 \mathrm{sec}$; while the flash power was manually adjusted to enable sufficient exposure of the image, typically between $1 / 16-1 / 32$ power. The resolution at $5 x$ was 1.212 pixels/ $\mu \mathrm{m}$ for the DSLRStackShot system compared with 1.135 pixels/ $/ \mathrm{m}$ for the automated slide scanner. As a result, there was no noticeable difference in the image resolution between these two systems (Figure 4).
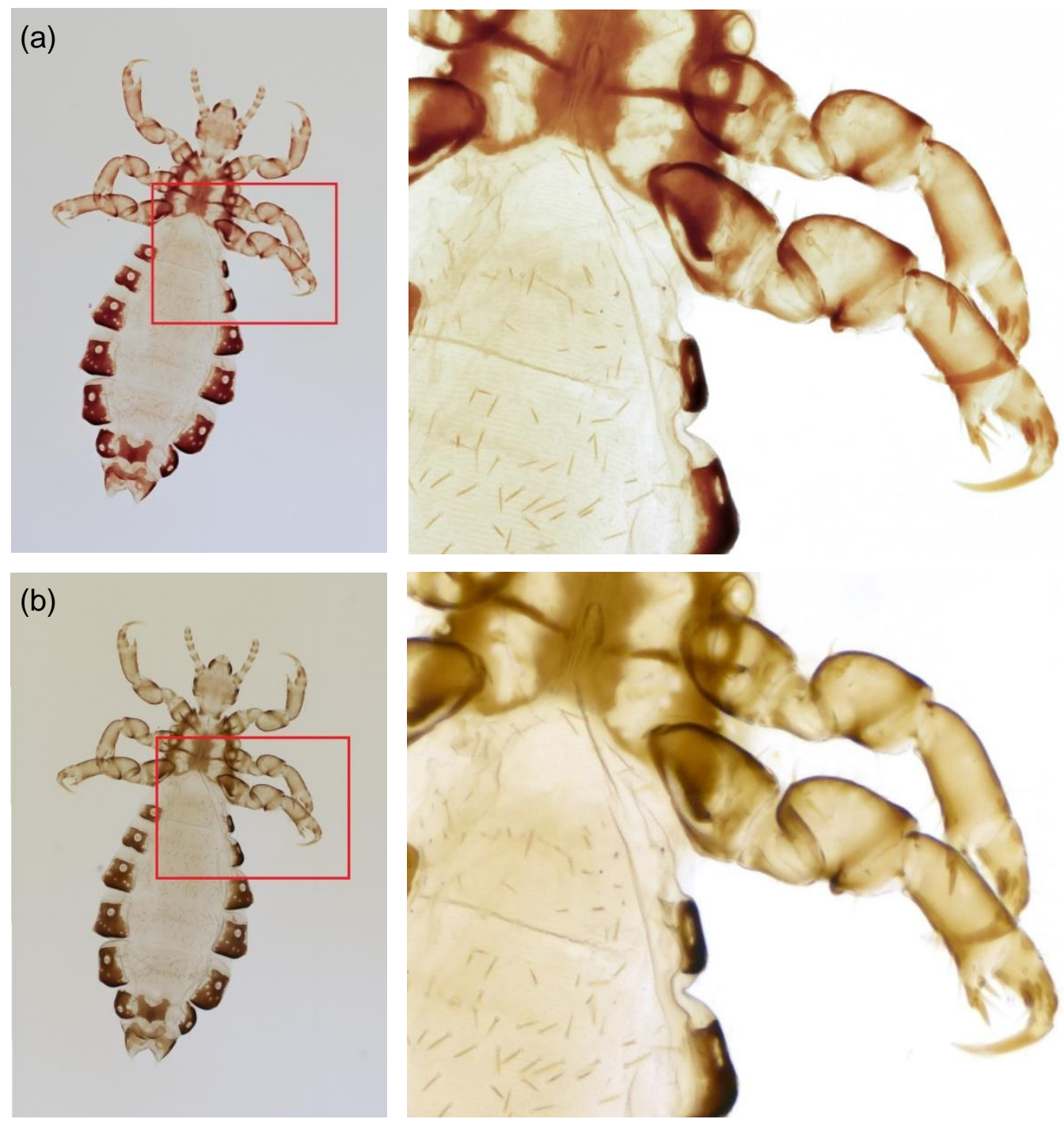

Figure 4. Image resolution comparison at $5 x$ magnification between (a) the DSLR-StackShot system and (b) an automated slide scanner. Specimen: human head louse. Left column: overview of the specimen; right column: highlighted box at $100 \%$ view. 


\section{Software and workflow}

The camera and Stackshot were controlled with Helicon Remote v.3.8.4 W (https://www.heliconsoft.com/heliconsoft-products/helicon-remote/). For each slide the top and bottom of the specimen were set in Helicon Remote and a suitable number of images are calculated based on a predefined interval to enable sufficient focal overlap for downstream image stacking. Each set of images was saved to a new folder, which was renamed with the specimen's unique identifier (UID). At the end of the day the images were stacked as a batch using Helicon Focus v.6.7.1 (https://www.heliconsoft.com/heliconsoft-products/helicon-focus/). The stacked images were then renamed in bulk with their corresponding folder name (UID) using Bulk Rename Utility v.3.0.0.1 (https://www.bulkrenameutility.co.uk/Download.php), and the scale bar was stamped using an ImageJ v.1.52h macro within Fiji (https://imagej.net/Fiji/Downloads).

Unlike automated slide scanners the addition of scale bars for the DSLR-StackShot system is a manual step and requires new scale bars to be calculated if the magnification changes. To minimise this manual step, images were captured at the same (maximum) magnification, thus facilitating batch processing. If the specimen was larger than the field of view at maximum magnification ( $5 \times$ field of view $=7 \times 5 \mathrm{~mm}$ ) then a lower magnification, and new scale bar, was required. Where possible these specimens were imaged in batches to facilitate downstream automation. Adaptations to this workflow are currently being investigated for more automated ways to identify the lens magnification via image EXIF data and apply the associated scale.

\section{Specimen Level Imaging of Natural History Slides}

During the two DCP slide digitisation projects a subset of slides were selected for specimen level imaging using an automated slide scanner. Some slides, however, were unsuitable for imaging using an automated system, despite the adaptations, and were imaged using the DSLR-StackShot system. Below we list a number of issues common to natural history slides that hindered the use of currently available automated slide scanners.

\section{Slide suitability}

Variation in size and thickness of slides is common among natural history collections. In order to image larger slides using the automated slide scanner, ZEISS created a variety of slide holders, known as magazines; however, some slides were too irregular or delicate to be placed in these magazines. With the DSLR-StackShot system, as the slide can be place 'freely' on the flashbox within the field of view it can accommodate any size of slide as well as broken slides. Most importantly, however, is the thickness of the mountant and coverslip (Figure 1) as there needs to be sufficient space between the lens and the slide to prevent damage. With the DSLR-StackShot system this is not an issue as there is a $40 \mathrm{~mm}$ working distance between the lens and the stage (Figure 3). 


\section{Failed to autofocus on specimen}

Slide scanners are highly automated, unlike the DSLR-StackShot system; however, these automated processes, in particular the autofocus, can be problematic when imaging historical slide collections.

i) For some slides, an automated slide scanner may fail to locate and focus on the specimen during the scanning process because $(A)$ the mountant is too thick and the specimen is positioned out of the focal range for the scanning profile, $(B)$ the specimen has not been stained sufficiently so there is little to no contrast to enable detection of the specimen, referred internally as a 'ghost' specimen, or (C) the mountant is too dark and the specimen cannot be detected, especially problematic for slides where the mountant is degrading. If a specimen cannot be located the slide scanner will skip the slide, flag that an error has occurred, and move the next slide into position for imaging. During the 2015 project, 4\% of the Entomological slides from Sternorrhyncha, Psocoptera and Phthiraptera resulted in this automated error. As a result, during the 2015 and 2017 projects any slides that fell within one of the three categories mentioned above were not selected for imaging using the automated slide scanner.

ii) For other slides an image was captured but the specimen was out of focus, thus at the end of each run all images had to be quality checked for focus issues or stitching errors. Natural history slides are likely to require cleaning prior to imaging using autofocus to ensure that the slide scanner does not focus on the dust on the coverslip. This was an important but time consuming process, which can be problematic for slides that are delicate or have markings on the coverslip that should not be erased. Other slides where autofocus was an issue included those with cracks in the cover slip, crystallisation of the mountant, air bubbles / foreign particles in the mountant, or markings on the coverslip i.e. a dot or arrow to indicate a particular specimen (Figure 5). These slides can be reimaged but there is a high probability that the autofocus will fail to focus on the specimen again. With the DSLR-StackShot system all the images were guaranteed to be in focus as the operator manually set the focal range for each specimen. 
(a)

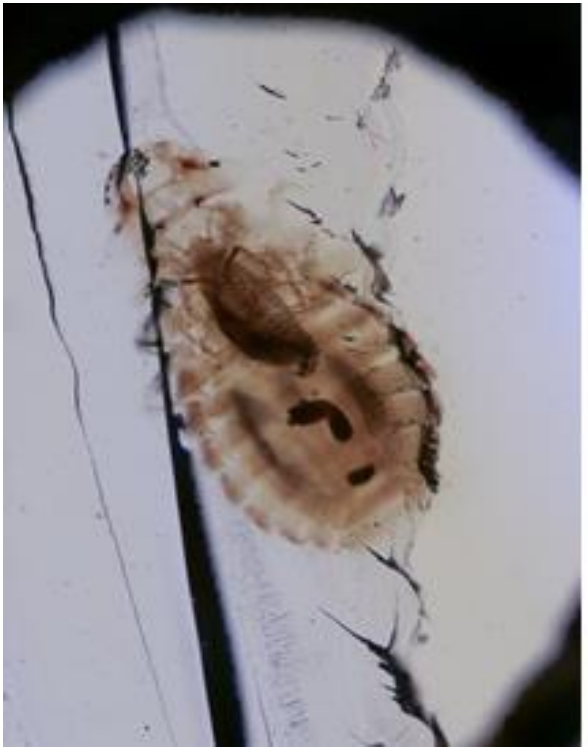

(c)

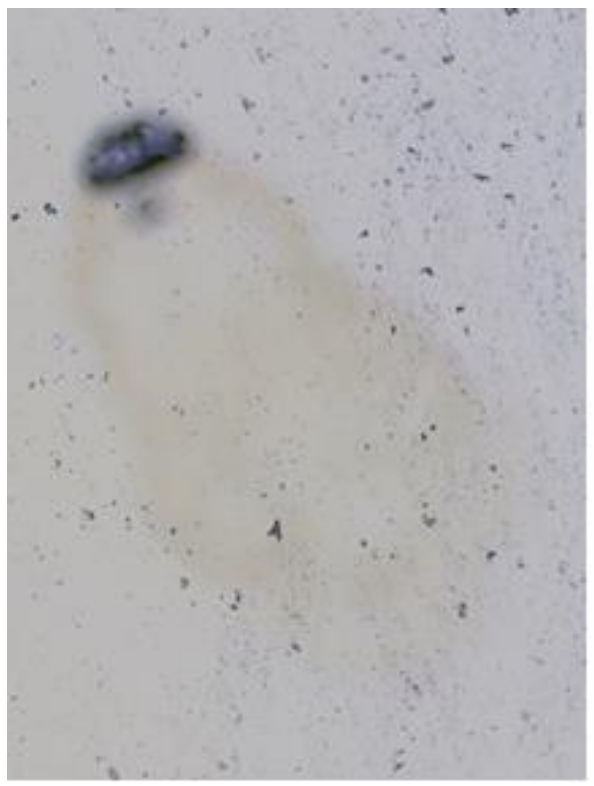

(b)

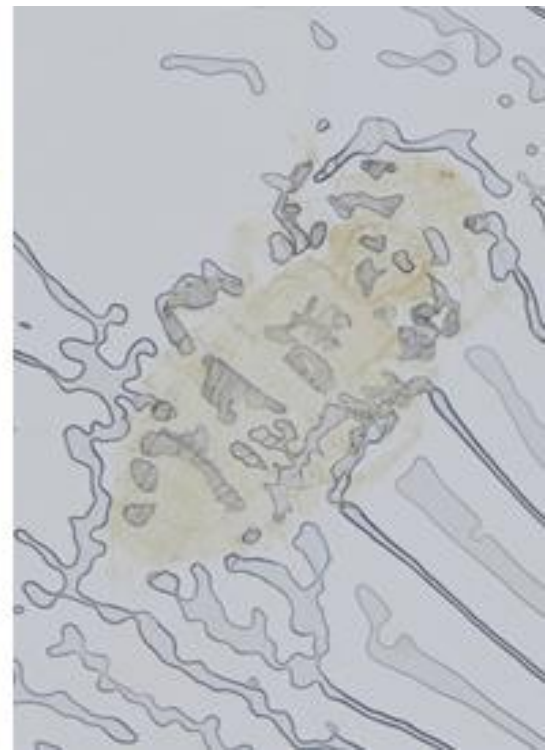

(d)

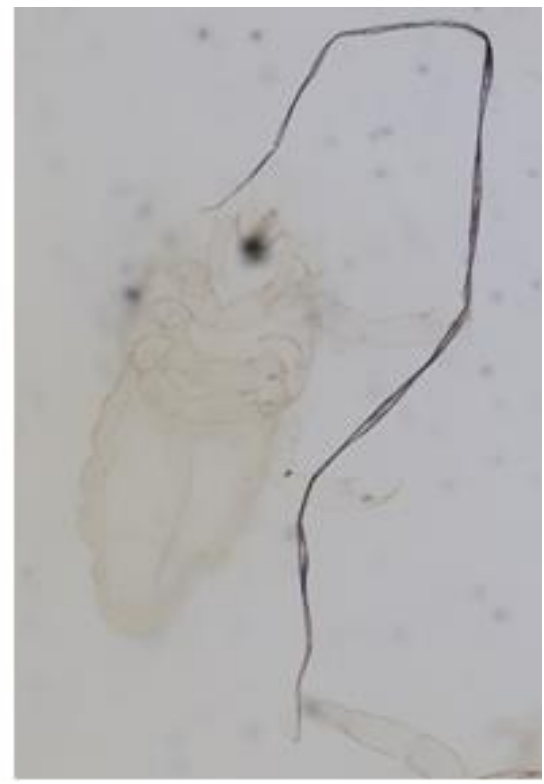

Figure 5. Examples of images taken using an automated slide scanner where the specimens are out of focus for the following reasons: (a) cracks in the slide, (b) crystallisation of the mountant, (c and d) foreign particles in the mountant.

\section{Specimen partially in focus}

In some instances part of the specimen was out of focus. This error normally occurred if the specimen was large or had extremities that exceeded the focal range setup for the scanning profile, or if there were multiple specimens at different heights in the mountant (Figure 6). To correct for these partial focus issues for large specimens the focal range of the scanning profile can be expanded, but this will increase the imaging time for each specimen. As you manually set the focal range when using the DSLR-StackShot system, you can adjust the range for each specimen thus enabling flexibility if you are imaging highly variable specimens. 

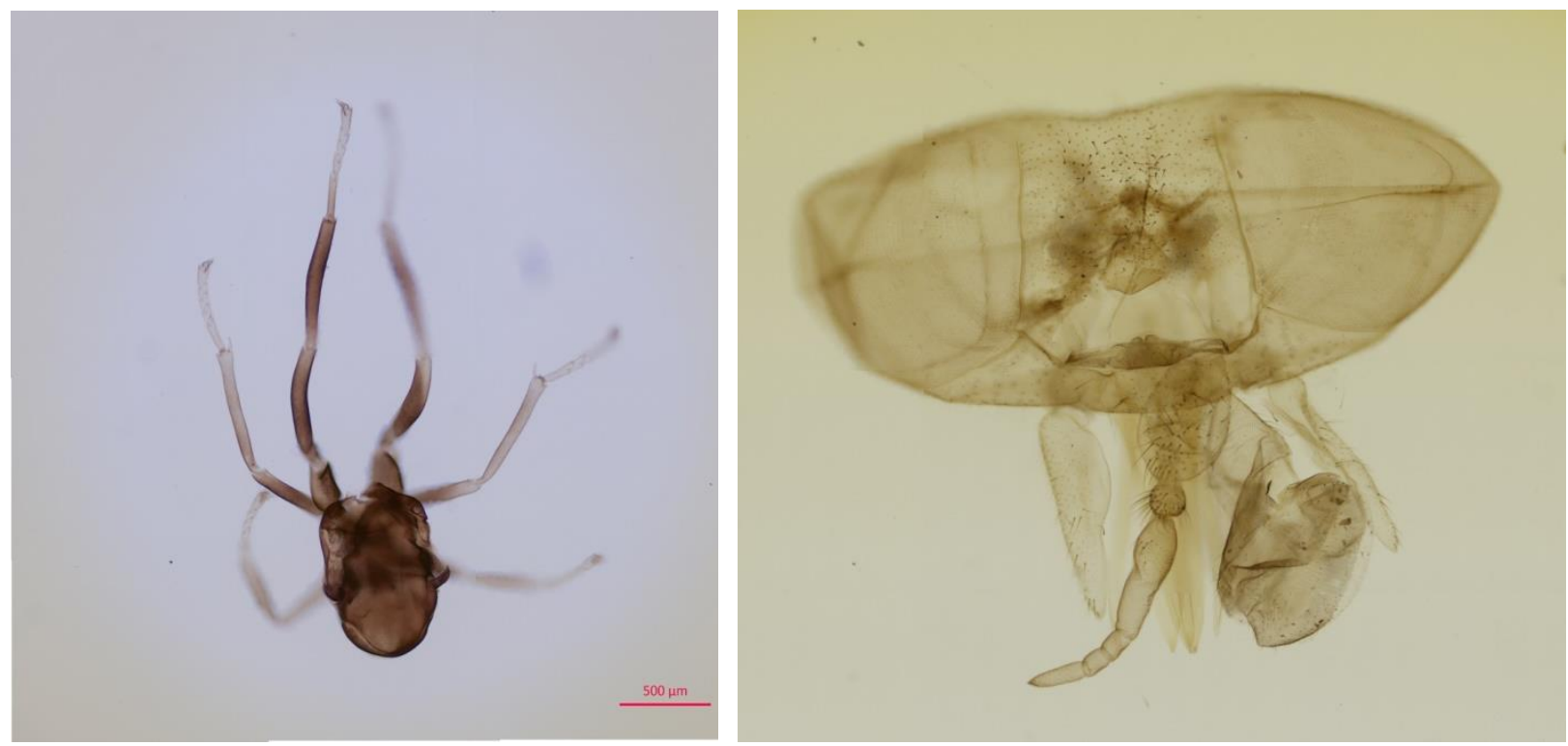

Figure 6. Examples of images taken using an automated slide scanner where part of the specimen is out of focus as it extends beyond the focal range of the scanning profile.

\section{Summary}

Natural history slides, due to their varied nature, are not always suitable for the currently available automated slide scanners. During the 2017 DCP Phthiraptera slide digitisation project we modified our DSLR imaging setup to be able to carry out specimen level imaging of non-uniform slides at $5 x$ lifesize magnification. While slide scanners can increase the imaging efficiency through automated processes, the DSLR-StackShot can provide additional flexibility for imaging slides of varying sizes, thickness, preservation types, as well as deteriorating and broken slides. This system also provides more external control during imaging, which is particularly useful for variable sized specimens and poor quality slides where autofocus is problematic. While there are limitations with more manual systems, such as the manual addition of scale bars, the DSLR-StackShot is a flexible, low cost alternative for imaging non-uniform natural history slides.

\section{Acknowledgements}

We thank the following people for assistance during the DCP slide digitisation projects: Rebecca Summerfield, Vladimir Blagoderov, Paul Brown, Charlotte Barclay, and the ZEISS support team.

\section{Funding program}

This research received support from the Museum's Digital Collection Programme and the SYNTHESYS Project (http://www.synthesys.info/), which is financed by the European Community Research Infrastructure Action under the FP7 Integrating Activities Programme. This paper was written as part of our contribution to ICEDIG - "Innovation and consolidation for large scale digitisation of natural heritage" (https://icedig.eu/) - an H2020 funded design study to supporting the implementation phase the DiSSCo research infrastructure (http://dissco.eu/). 


\section{Grant title}

SYNTHESYS Project (Grant agreement number 312253) and ICEDIG (Grant agreement number 777483).

\section{References}

Allan EL, Livermore L, Price BW, Shchedrina O, Smith VS (2019) A novel automated mass digitisation workflow for natural history microscope slides. Biodiversity Data Journal, e32342. https://doi.org/10.3897/BDJ.7.e32342

Decker P, Christian A, Xylander WE (2018) VIRMISCO - The Virtual Microscope Slide Collection. In: Stoev P, Edgecombe GD (Eds) Proceedings of the 17th International Congress of Myriapodology, Krabi, Thailand. ZooKeys, 741, 271-282. https://doi.org/10.3897/zookeys.741.22284

Dietrich C, Hart J, Raila D, Ravaioli U, Sobh N, Sobh O, Taylor C (2012). InvertNet: a new paradigm for digital access to invertebrate collections. ZooKeys 209: 165-181. https://doi.org/10.3897/zookeys.209.3571

Drew JA, Moreau CS, Stiassny MLJ (2017) Digitization of museum collections holds the potential to enhance researcher diversity. Nature Ecology \& Evolution 1 (12): 1789-1790. https://doi.org/10.1038/s41559-017-0401-6

Heerlien M, Van Leusen J, Schnörr S, de Jong-Jole S, Raes N, van Hulsen K (2015) The natural history production line: an industrial approach to the digitization of scientific collections. ACM Journal on Computing and Cultural Heritage 8: 11 pages. http://dx.doi.org/10.1145/2644822

Musson A, Reed L, Bojarska M, Fulcher T (2015) Digitising Kew's microscope slide collection. https://www.kew.org/blogs/kew-science/digitising-kews-microscope-slide-collection. Accessed on: 2018-06-08.

Rojo MG, García GB, Mateos CP, García JG, Vicente MC (2006) Critical comparison of 31 commercially available digital slide systems in pathology. International Journal of Surgical Pathology 14 (4): 285-305. https://doi.org/10.1177/1066896906292274

Summerfield R, Sherlock E, Price BW, Allan EL, Ball A, Shchedrina O, Arnold N, Blagoderov V (in prep) An approach to high throughput slide digitisation using image segmentation. OSF Preprints.

Weinstein RS, Graham AR, Richter LC, Barker GP, Krupinski EA, Lopez AM, Erps KA, Bhattacharyya AK, Yagi Y, Gilbertson JR (2009) Overview of telepathology, virtual microscopy, and whole slide imaging: prospects for the future. Human Pathology 40 (8): 1057-1069. https://doi.org/10.1016/j.humpath.2009.04.006 